\title{
Fragmentación de pastizales psamófilos por plantaciones de pinos: efectos sobre la riqueza y la composición vegetal
}

\author{
Alejandra L. YezZi ${ }^{1}{ }^{\Perp} ;$ Ana J. Nebbia ${ }^{1} \&$ Sergio M. Zalba ${ }^{1}$ \\ ${ }^{1}$ GEKKO - Grupo de Estudios en Conservación y Manejo, Departamento de Biología, Bioquímica y Farmacia, Universidad \\ Nacional del Sur. Bahía Blanca, Buenos Aires, Argentina.
}

\begin{abstract}
Resumen. Las dunas litorales de la Pampa Austral se encuentran mejor conservadas que el resto de los pastizales pampeanos, pero igualmente presentan amenazas derivadas de actividades antrópicas. La forestación con especies exóticas reduce la superficie del pastizal psamófilo, fragmentando el ecosistema y limitando la vegetación original a áreas relictuales. Los objetivos de este trabajo fueron evaluar los efectos de la fragmentación sobre las comunidades vegetales e identificar parámetros que ponen en evidencia los impactos de este proceso con mayor claridad. Se evaluaron atributos comunitarios en fragmentos de pastizal de distinto tamaño ( 0.05 a 2.5 ha) inmersos en una matriz forestal y se los comparó con áreas control de pastizal continuo de superficie equivalente. Se encontró una relación positiva entre la superficie de las unidades muestrales y la riqueza de plantas nativas y exóticas, y la diversidad total. Los fragmentos menores presentaron mayor riqueza de exóticas y menor riqueza de nativas respecto al pastizal continuo. Entre las especies predominantes en fragmentos menores se hallaron Ambrosia tenuifolia, planta nativa asociada a disturbios, y la exótica Senecio madagascariensis, y en fragmentos mayores las nativas Cyperus reflexus e Hydrocotyle bonariensis, características de áreas húmedas. En el pastizal continuo predominaron gramíneas y otras nativas típicas del pastizal pampeano, como Panicum urvilleanum y Margyricarpus pinnatus. La composición específica resulta un indicador más preciso de las alteraciones asociadas a la fragmentación del pastizal comparándolo con los cambios en la riqueza y diversidad de especies, y permite plantear hipótesis acerca de factores particulares que podrían explicar la presencia o abundancia de determinados taxones. Las variaciones en la representación de ciertos grupos pueden orientar trabajos dirigidos a evaluar el efecto de la fragmentación sobre diferentes procesos ecológicos.
\end{abstract}

[Palabras clave: destrucción de hábitats, bioma pampeano, dunas litorales, plantas exóticas invasoras]

\begin{abstract}
Aвstract. Psamophilous grassland fragmentation due to pine plantations: effects on species richness and plant composition. The coastal dunes of the Pampa Austral are comparatively better preserved than the rest of the Pampas grasslands, but they are also threatened by anthropogenic intervention. Plantations of exotic species reduce the area of psamophilous grasslands, fragmenting the ecosystem and limiting the original native vegetation to relic areas. The objectives of this study were to evaluate the effects of grassland fragmentation on plant communities and to identify parameters that highlight the impacts of this process with greater clarity. Community attributes were evaluated in grassland fragments of different size ( 0.05 to $2.5 \mathrm{ha})$ surrounded by a forest matrix and compared to control areas of continuous grasslands of equivalent area. A significant positive relationship was found between the area of the study units and the richness of native and exotic plants, and total plant diversity. The smaller fragments presented greater exotic species richness and less native species richness than the continuous grasslands. Ambrosia tenuifolia, a native species associated with disturbances, and the exotic Senecio madagascariensis, were found among the predominant species in smaller fragments. In the larger fragments predominated the native Cyperus reflexus and Hydrocotyle bonariensis, all characteristic of humid habitats. Grasses and other native plants, such as Panicum urvilleanum and Margyricarpus pinnatus, predominated in the continuous grasslands. We found species composition to be a more sensible indicator of the alterations associated with the fragmentation of grasslands when compared with changes in species richness and diversity, and it also allows to hypothesize about particular factors that could explain the presence or abundance of certain taxa. Variations in the distribution of certain taxonomic groups can guide research aimed at evaluating the effect of fragmentation on different ecological processes .
\end{abstract}

[Keywords: habitat destruction, Pampa biome, coastal dunes, invasive alien plants]

Editor asociado: Ricardo Grau

$\bar{\triangle}$ alejandrayezzi@gmail.com
Recibido: 18 de agosto de 2017

Aceptado: 29 de noviembre de 2017 


\section{INTRODUCCIÓN}

La fragmentación de ambientes naturales es uno de los principales procesos responsables de la crisis global de pérdida de biodiversidad (Haddad et al. 2015; Wilson et al. 2016), afectando los ambientes naturales a través del aislamiento de los fragmentos generados y de la influencia de la matriz desde los bordes resultantes. Estos cambios se traducen en alteraciones en la abundancia y riqueza de las especies, reducción del tamaño de las poblaciones, pérdida de diversidad genética y modificaciones en la estructura del paisaje, entre otros efectos (Saunders et al. 1991; Haddad et al. 2015; Wilson et al. 2016). Numerosos estudios han cuantificado estos impactos, aunque la mayoría se concentraron en ambientes de bosque templado y tropical (Thiollay 1988; Benitez 1998; Barnes et al. 2014; entre otros), mientras que mucho menos se sabe acerca de los efectos de la fragmentación sobre otros ecosistemas, como las estepas y los pastizales. Los pastizales se reconocen como el bioma terrestre donde la biodiversidad y los servicios ecosistémicos están en mayor riesgo a escala mundial, debido a la gran disparidad entre la tasa de pérdida de hábitat y el grado de protección efectiva (Hoekstra et al. 2005; Suttie et al. 2005). Los pastizales del sureste de América del Sur, en particular, han experimentado un proceso de transformación y fragmentación debido al avance de diversas formas de intervención antrópica (Bilenca and Miñarro 2004; Azpiroz et al. 2012). La sustitución de pastizales naturales por plantaciones forestales se destaca entre ellas (Guadagnin et al. 2009; Simberloff et al. 2010; Fonseca et al. 2013). Esta expansión ha estado incentivada por la posibilidad de lograr altos rendimientos de biomasa en tierras de pastizal y por las perspectivas del inminente comercio de bonos de carbono (Jobbágy et al. 2006). Las transiciones entre sistemas dominados por pastos a sistemas dominados por árboles suelen tener un gran impacto sobre el funcionamiento de los ecosistemas, como resultado del contraste entre estos dos grandes grupos de plantas en relación a la utilización de la energía, el agua y los nutrientes (Raffaele and Schlichter 2000), y por ende sobre la provisión de ciertos bienes y servicios ecosistémicos, como el mantenimiento de la biodiversidad, el secuestro de carbono, recursos genéticos, conservación del suelo y regulación del ciclo hidrológico (Costanza et al. 1997; Baldi et al. 2006).
En Argentina, la región pampeana se encuentra entre los biomas con mayores niveles de degradación y es, a su vez, una de las que menor atención ha recibido desde el punto de vista conservacionista, contando con uno de los niveles más bajos de protección (Burkart 2006). La superficie de pastizales en buen estado de conservación está restringida a ambientes donde la frontera agropecuaria no ha podido avanzar, como los afloramientos rocosos, los humedales y las dunas de la franja costera (Zalba and Villamil 2002; Baldi et al. 2006). Si bien existen menciones acerca de los efectos negativos causados por la fragmentación en ambientes de pastizal pampeano (Campo et al. 1999; Bilenca and Miñarro 2004), son pocos los estudios que cuantifiquen objetivamente los efectos de este proceso, entre ellos Cairo y Zalba (2007), Herrera y Laterra (2007), Nebbia y Zalba (2007), y Lara y Gandini (2011, 2014). Entre sus consecuencias se destaca, no sólo un aumento del riesgo de extinción de especies nativas, sino también la conversión de estos relictos de pastizal pampeano en ambientes más vulnerables a las invasiones por especies exóticas, lo que transforma a la fragmentación en un proceso potenciador de otras alteraciones antrópicas (With 2004; Yates et al. 2004).

La capacidad de los pastizales fragmentados para sostener la biodiversidad y los servicios ecosistémicos dependerá de la cantidad y calidad del hábitat en los remanentes, de su grado de conectividad, y de cómo se vean afectados por otras perturbaciones inducidas por el hombre, tales como el cambio climático o las especies invasoras (Wilson et al. 2016). Estudiar los efectos de la fragmentación sobre la biodiversidad resulta fundamental para tomar decisiones de manejo orientadas a reducir, mitigar o compensar el impacto de este proceso, para ello es muy importante determinar la susceptibilidad de distintos niveles de organización ecológica frente a la fragmentación del hábitat (Haddad et al. 2015). Tradicionalmente los estudios que se han desarrollado para evaluar los efectos de la fragmentación sobre la biodiversidad están centrados en sus impactos sobre la riqueza y diversidad de las especies presentes (Fahrig 2003; Bieringer et al. 2013; Krause et al. 2015). Sin embargo, distintos autores sostienen que una comprensión más completa del fenómeno de fragmentación y de sus consecuencias requiere ampliar el análisis, ya que otras medidas de la estructura comunitaria, tales como la 
composición específica, la organización trófica y la abundancia relativa de los distintos grupos funcionales, podrán informar mejor sobre cómo la fragmentación afecta a las comunidades bióticas, incluso cuando la riqueza de especies per se no resulte alterada (Metzger 2000; Halffter and Moreno 2005; Schmidt and Roland 2006; Haddad et al. 2015; Wilson et al. 2016).

Las dunas costeras de la provincia de Buenos Aires constituyen ambientes de gran valor, presentando una alta riqueza biológica y ambiental, y brindando importantes servicios ecosistémicos (Kittlein et al. 2004; Celsi and Monserrat 2006). Sin embargo, distintas actividades antrópicas fragmentan y modifican el ambiente natural (Dadon 2002; Monserrat and Celsi 2009), principalmente el establecimiento y expansión de núcleos urbanos y la forestación con especies exóticas (Guadagnin et al. 2009; Azpiroz et al. 2012).

Este trabajo analiza los efectos de la fragmentación de pastizales como consecuencia de la forestación con Pinus pinaster (pino marítimo), una especie con comportamiento invasor en Argentina y Uruguay (Picasso and Rodríguez 2008; Brugnoli et al. 2009; Cuevas and Zalba 2009). Se evalúan las alteraciones en distintos parámetros de la estructura comunitaria en un sector de pastizal psamófilo en el sudoeste de la provincia de Buenos Aires. Proponemos que, si bien la riqueza y la diversidad de especies vegetales mostrarán una relación directa con el área, tanto en fragmentos rodeados por pinos como en pastizales continuos, las dos variables resultarán mayores en el pastizal no fragmentado cuando se comparen superficies de igual tamaño. Esperamos, además, que la proporción de especies nativas oportunistas y exóticas, resulte mayor en los fragmentos rodeados por la forestación.

\section{Materiales y MÉTOdos}

\section{Área de estudio}

Las dunas del sur de la provincia de Buenos Aires (barrera medanosa austral) se ubican en el extremo sur de la Provincia Pampeana, lindante hacia el oeste con la Provincia del Espinal (Cabrera 1971). El clima es templado, influenciado por la cercanía al mar (Campo de Ferreras et al. 2004). La franja costera varía entre 2 y $7 \mathrm{~km}$ de ancho, incluyendo las playas, médanos vivos, médanos fijos (con pastizales psamófilos) y depresiones intermedanosas (pastizales húmedos y salinos). Sobre las dunas predominan las comunidades psamófilas de Hyalis argentea, Panicum urvilleanum, Thelesperma megapotamicum y Oenothera sp. (Zalba and Nebbia 1999). En las depresiones intermedanosas se encuentran cortaderales (Cortaderia selloana), juncales (Juncus acutus y Schoenoplectus americanus) y totorales (Typha latifolia) (Celsi and Monserrat 2008). Se destacan endemismos locales como Neosparton darwinii, Noticastrum sericeum y Senecio bergii, entre la flora, y el tuco-tuco de las dunas (Ctenomys australis) y la lagartija de las dunas (Liolaemus multimaculatus) entre la fauna. Además el área se superpone, en parte, con la zona de invernada del amenazado cauquén colorado (Chloephaga rubidiceps) (Kittlein et al. 2004).

El área estudiada (costa del Partido de Coronel Dorrego) posee extensas superficies de pastizales naturales caracterizados por una alta riqueza biológica y ambiental (Celsi and Monserrat 2008). El nivel de modificación antrópica es muy bajo (Monserrat and Celsi 2009), pudiéndose mencionar la actividad forestal y el uso agropecuario asociado a la presencia de ganado vacuno en algunos sectores. La superficie forestada se concentra principalmente dentro y en los alrededores de los límites urbanos, y se trata de plantaciones puntuales y aisladas (Celsi and Monserrat 2006). La masa forestal principal corresponde a una plantación de pino marítimo (Pinus pinaster) que cubre una superficie total aproximada de 30 ha (Figura 1).

\section{Diseño de muestreo}

A partir de una imagen satelital del sitio tomada de Google Earth $₫$ y fechada el 14 de diciembre de 2006 se identificaron remanentes de vegetación natural inmersos en el área forestada con pino marítimo y se midió la superficie y el perímetro de cada uno de ellos utilizando las herramientas de Google Earth Pro ®. Los fragmentos fueron individualizados y validados a campo, confirmando que no se hubieran producido cambios en su superficie y forma desde la captura de la imagen satelital. Se seleccionaron tres fragmentos de pastizal en cada una de cinco categorías de tamaño: hasta 0.05 ha, de 0.05 a 0.1 ha, de 0.1 a 0.5 ha, de 0.5 a 1 ha y de 1 a 2.5 ha, totalizando quince unidades muestrales. Los controles de pastizal continuo se ubicaron en áreas vecinas sin forestación. Utilizando rumbos y distancias 


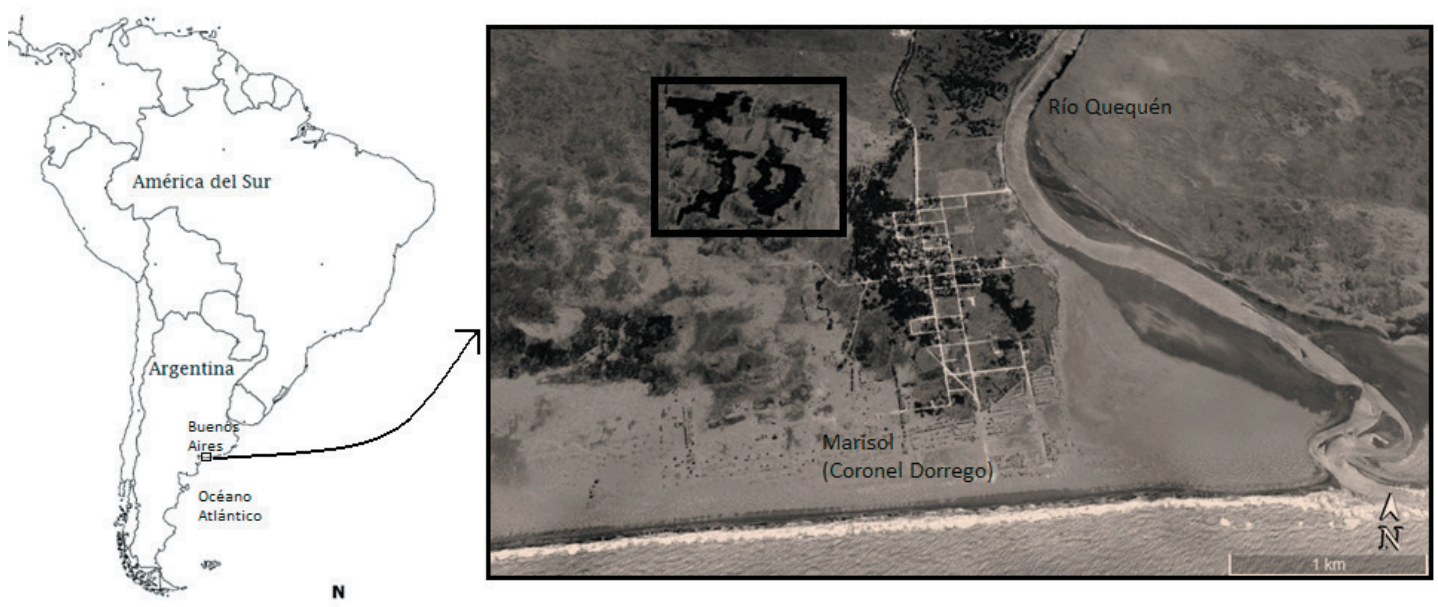

Figura 1. Ubicación del área de estudio en el sudoeste de la Provincia de Buenos Aires, Argentina, indicando la forestación de pino marítimo en un sector de dunas fijas junto a la localidad de Marisol, en el Partido de Coronel Dorrego $\left(38^{\circ} 55^{\prime} \mathrm{S}, 60^{\circ} 33^{\prime} \mathrm{O}\right)$.

Figure 1. Location of the study area in the southwest of Buenos Aires Province, Argentina, indicating the distribution of Pinus pinaster plantations in fixed dunes next to the town of Marisol, at Coronel Dorrego County $\left(38^{\circ} 55^{\prime} \mathrm{S}, 60^{\circ} 33^{\prime}\right.$ $\mathrm{W})$.

seleccionadas de una tabla de números al azar se establecieron 15 parcelas, tres para cada una de las categorías de tamaño definidas. A partir de los datos de área y perímetro se calculó un índice de forma (IF) para cada fragmento:

$$
\mathrm{IF}=(\sqrt{\mathrm{A}}) /(0.282 * \mathrm{P})
$$

donde A es el área, $\mathrm{P}$ el perímetro y la constante 0.282 corrige la fórmula de modo que IF $=1$ para formas circulares (Nebbia and Zalba 2007).

En las treinta unidades muestrales definidas se realizaron censos de vegetación siguiendo el método de relevés de Braun Blanquet (Mueller Dombois and Ellenberg 1974) para estimar el porcentaje de cobertura por especie. Los ejemplares vegetales fueron determinados a nivel de especie y/o género (Cabrera 19631970) y se clasificaron en especies nativas y exóticas. Para la nomenclatura se empleó el Catálogo de las Plantas Vasculares del Cono Sur (Zuloaga et al. 2008).

\section{Análisis de datos}

Se registraron la riqueza y la proporción de especies nativas y exóticas, y el índice de diversidad de Shannon (Begon et al. 2006) para cada tamaño, tanto en los fragmentos rodeados por forestación como en el ambiente continuo. Se evaluó la normalidad de los datos y se verificó la homocedasticidad a partir del estudio de residuos y de gráficos de probabilidad normal, se realizaron transformaciones a los datos que no cumplían con los supuestos arriba mencionados.

Riqueza específica. Se construyeron modelos lineales generalizados con una distribución de Poisson para analizar el posible efecto de la superficie de las unidades de estudio y del contexto (matriz forestal o pastizal continuo) sobre la riqueza de especies nativas y sobre la riqueza de especies exóticas. Los modelos generados se compararon a través de un Likelihood Ratio Test (LRT). La riqueza de especies nativas y exóticas entre fragmentos y áreas continuas, dentro de cada categoría de superficie, se compararon mediante test de ANOVA de una vía. Se realizaron regresiones utilizando modelos lineales generalizados con una distribución de Poisson entre los valores de riqueza de especies nativas y de riqueza de especies exóticas, y análisis de regresión lineal simple para los residuales de los análisis riqueza-área respecto del índice de forma de los fragmentos (IF).

Diversidad. Se construyeron modelos lineales para analizar el posible efecto de la superficie de las unidades de estudio y el contexto (matriz forestal o pastizal continuo) sobre la diversidad de especies. Los modelos generados se compararon a través de un ANCOVA. Se aplicaron transformaciones a las variables para cumplir con los supuestos de normalidad y homocedasticidad (logaritmo decimal para área). Se realizaron análisis de regresión lineal simple entre los valores de diversidad y los residuales del análisis 
diversidad-área respecto del índice de forma de los fragmentos (IF).

Composición. Para determinar si existía algún tipo de agrupamiento de las muestras en función de su composición específica y si este agrupamiento estaba correlacionado con la superficie y/o con el contexto de las unidades muestrales, se realizó un ACP (Análisis de Componentes Principales) a partir de la matriz de covarianza de los datos de cobertura de las especies. Para mantener un balance apropiado entre el número de muestras y de variables (cobertura por especie) se excluyeron del análisis las plantas menos abundantes (aquellas presentes en menos de nueve muestras, $55.8 \%$ del total de las especies)

Todos los análisis y los gráficos se realizaron utilizando los paquetes estadísticos Infostat y XLSTAT.

\section{Resultados}

Se registró la presencia de un total de 68 especies pertenecientes a 20 familias. Cincuenta y cuatro de esas especies eran nativas y 14 exóticas. Las familias mejor representadas fueron Asteraceae (38\%) y Poaceae $(24 \%)$. Ambos sitios, fragmentos de pastizal rodeados por la forestación y controles de pastizal continuo, contaron con un número similar de especies totales (57 y 56 , respectivamente), con 45 especies comunes. La riqueza total de especies fue de 28, 38, 33, 39 y 42 para los fragmentos de $0.05,0.1,0.5$,

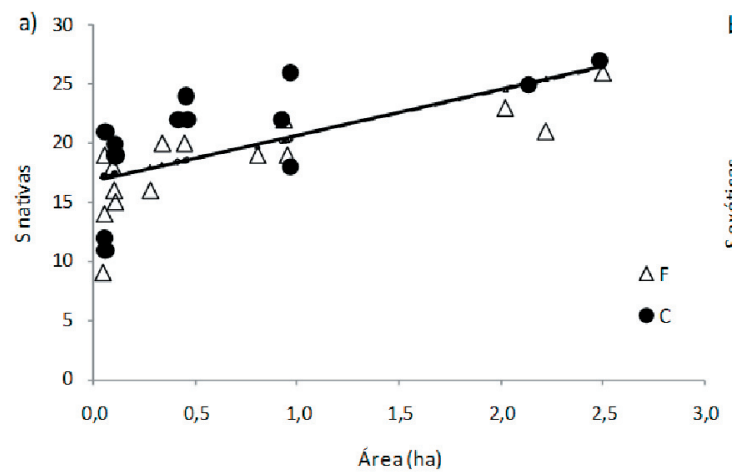

1 y 2.5 ha rodeados por la matriz forestal, respectivamente. Los controles presentaron una mayor proporción total de especies nativas (84\%) que los fragmentos (75\%). La especie endémica Baccharis divaricata se registró con mayor frecuencia en los controles (40\%) que en los fragmentos (13\%). Pinus pinaster, especie constituyente de la matriz forestal, se registró con mayor frecuencia en los fragmentos (47\%) que en los controles (7\%), presentando baja cobertura promedio en ambos contextos ( 0.15 y $0.01 \%$, respectivamente) (ver Material suplementario).

\section{Riqueza}

La superficie de las unidades muestrales resultó un buen predictor de la riqueza de especies nativas $\left(X^{2}=15.82 ; P=0.0001\right)$, y no se detectó una interacción significativa con el contexto $(L R T=0.01 ; P=0.9238)$, por lo que se sugirió un único modelo para representar esta relación: $S$ nativas $=2.84+0.18$ *área (Figura 2a). La superficie mostró también una correlación significativa con la riqueza de especies exóticas $\left(X^{2}=13.31 ; P=0.0003\right)$. En este caso la interacción con el tipo de ambiente resultó estadísticamente significativa $(L R T=$ 5.94; $P=0.0148)$, definiéndose así un modelo para cada contexto ambiental: para fragmentos de pastizal rodeados por plantación de pinos (F), S exóticas $=1.54+0.20 *$ área; para controles de pastizal continuo $(\mathrm{C})$, S exóticas $=0.11+0.71$ * área (Figura 2b).

Se detectaron diferencias significativas entre ambos ambientes respecto a la riqueza

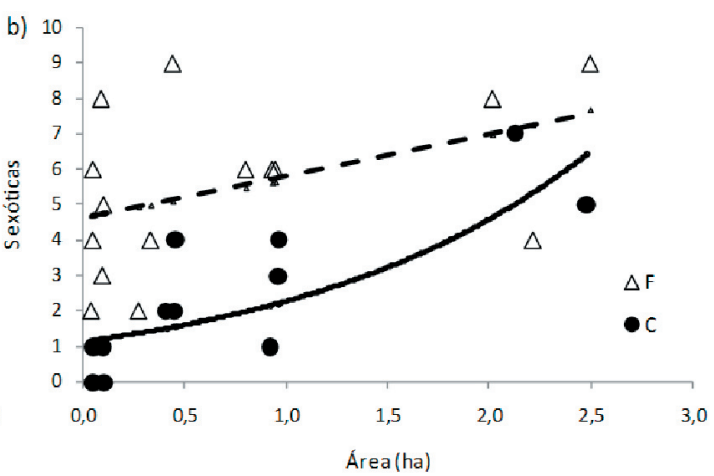

Figura 2. Relación entre riqueza de plantas nativas (a) y exóticas (b) y el área de fragmentos de pastizal rodeados por una plantación de pinos (F) y de controles de pastizal continuo de superficie equivalente (C) en dunas costeras del sudoeste bonaerense. Ambas relaciones representadas por un único modelo en el caso de las plantas nativas y por un modelo para fragmentos rodeados por pinos (línea discontinua) y otro para áreas rodeadas de pastizal (línea continua), en el caso de las plantas exóticas.

Figure 2. Relationship between species richness of native (a) and exotic plants (b) and the area of grassland fragments surrounded by a pine plantation $(F)$, and continuous grasslands controls of equivalent area (C) on coastal dunes of southwestern Buenos Aires Province. Both relationships represented by a unique model in the case of native plants, and by two models for exotic plants: one for the plots surrounded by pines (discontinuous line) and other for those in the grassland matrix (continuous line). 

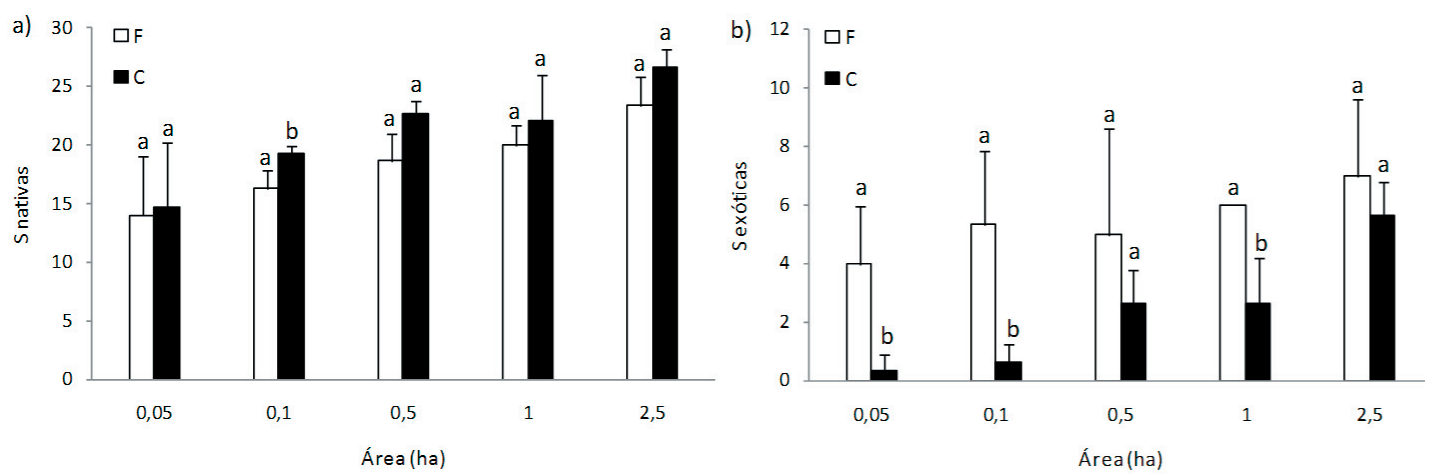

Figura 3. Comparación entre la riqueza promedio de especies nativas (a) y exóticas (b) en fragmentos de pastizal rodeados por plantación de pinos (F) y en controles de pastizal continuo de superficie equivalente (C) en dunas costeras del sudoeste bonaerense. Letras distintas indican diferencias significativas $(P<0.05)$, las barras representan el error estándar.

Figure 3. Comparison of the mean species richness of native (a) and exotic plants (b) in grassland fragments surrounded by a pine plantation $(\mathrm{F})$ and in controls of continuous grassland of equivalent area $(\mathrm{C})$ in coastal dunes of southwestern Buenos Aires Province. Different letters indicate significant differences $(P<0.05)$, bars represent the standard error.

promedio de especies nativas y de especies exóticas para unidades de muestreo de distinta superficie. Mientras que el número de nativas resultó significativamente mayor en áreas de pastizal continuo de 0.1 ha respecto de la de los fragmentos inmersos en la matriz forestal $(F=10.125 ; P=0.033$, Figura 3a), esta relación se invirtió en el caso del número de especies exóticas en áreas de $0.05(F=9.308 ; P=0.038)$, $0.1(F=9.800 ; P=0.035)$ y 1 ha $(F=14.286 ; P$ $=0.019$, Figura 3b).

El índice de forma de los fragmentos varió entre 0.38 y 0.79 . No se detectaron relaciones lineales significativas entre el índice de forma de los fragmentos y la riqueza de especies nativas y exóticas $\left(X^{2}=0.42 ; P=0.52\right.$ para nativas y $X^{2}=1.36 ; P=0.24$ para exóticas),

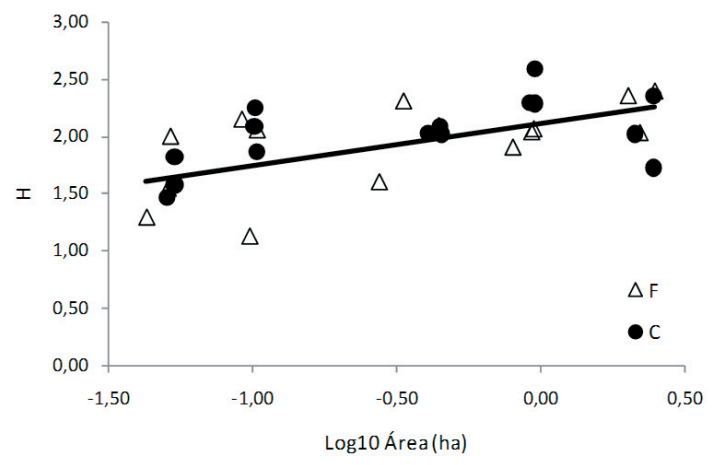

Figura 4. Relación entre la diversidad de especies vegetales y el área de fragmentos de pastizal rodeados por plantación de pinos (F) y de controles de pastizal continuo de superficie equivalente (C) en dunas costeras del sudoeste bonaerense.

Figure 4. Relationship between plant species diversity and area of grassland fragments surrounded by a pine plantation (F) and controls of continuous grasslands of equivalent area (C) in coastal dunes of southwestern Buenos Aires Province. ni respecto de los residuales de los análisis riqueza-área $(F=0.16 ; P=0.70$ para nativas y $F=0.65 ; P=0.44$ para exóticas).

\section{Diversidad}

La diversidad y el área de las unidades de estudioresultaronfuertementecorrelacionadas $\left(H=2.13+0.31{ }^{*} \log 10\right.$ área; $F=13.704 ;$ $P=0.001)$, y no se detectó una interacción significativa con el contexto ambiental $(T=$ $-0.625 ; P=0.538$, Figura 4 ).

Tampoco en este caso se detectaron relaciones lineales significativas entre el índice de forma de los fragmentos y la diversidad de especies $(F=0.04 ; P=0.85)$, ni en el análisis con los residuales diversidad-área $(F=0.07 ; P=$ $0.79)$.

\section{Composición}

Los dos primeros componentes del ACP explicaron un $51.40 \%$ de la varianza total. Sobre el primero (25.92\% de la varianza) se observa cierta separación entre las áreas menores ( 0.05 y 0.1 ha) y las áreas mayores (2.5 y 1 ha), estando la mayoría de estas últimas agrupadas en el tercer cuadrante (valores negativos de los dos componentes). Las áreas menores presentaron alta abundancia de las especies nativas Tessaria absinthioides, Oenothera mollissima y Conyza bonariensis, y de las especies exóticas Cirsium vulgare e Hypochaeris radicata. Las áreas mayores estuvieron asociadas con altas abundancias de las nativas Imperata brasiliensis, Solidago chilensis, Pseudognaphalium leucopeplum y Eragrostis airoides (Tabla 1, Figura 5a). El segundo componente ( $25.49 \%$ de la varianza) 

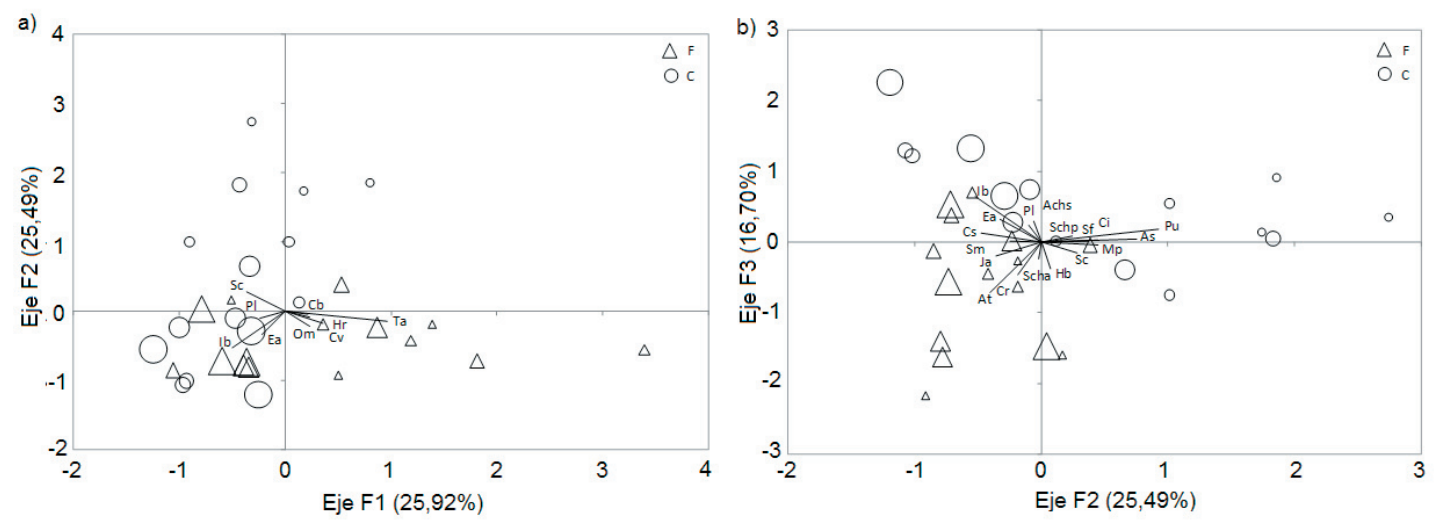

Figura 5. Análisis de Componentes Principales (ACP) desarrollado a partir de la matriz de covarianza de muestras de porcentaje de cobertura de especies vegetales en unidades de muestreo de distinta superficie ubicadas en sectores de pastizal continuo $(\mathrm{C})$ y rodeadas por plantación de pinos $(\mathrm{F})$ en dunas costeras del sudoeste bonaerense y vectores de las especies vegetales (ver referencias en la Tabla 1). (a) $1^{\circ} \mathrm{CP}$ y $2^{\circ} \mathrm{CP}$; (b) $2^{\circ} \mathrm{CP}$ y $3^{\circ} \mathrm{CP}$. Los símbolos de distinto tamaño representan unidades muestrales de distinta superficie (de 0.05 ha a 2.5 ha). Las abreviaturas de los nombres de las especies se incluyen en la Tabla 1.

Figure 5. Principal Components Analysis (PCA) developed from the covariance matrix of percent coverage of plant species in sampling units of different area located in continuous grasslands $(C)$ and surrounded by pine plantations (F) in coastal dunes of the southwestern Buenos Aires Province, and vectors of plant species (see references in Table 1). (a) $1^{\circ} \mathrm{PC}$ and $2^{\circ} \mathrm{PC}$; (b) $2^{\circ} \mathrm{PC}$ and $3^{\circ} \mathrm{PC}$. The different sizes of the symbols represent the area of the sampling units (from 0.05 ha to $2.5 \mathrm{ha}$ ). Species abbreviations are included in Table 1.

Tabla 1. Coeficientes de correlación entre la cobertura por especie y los tres primeros componentes del ACP en fragmentos de pastizal rodeados por plantación de pinos y controles de pastizal continuo de distinta superficie en dunas costeras del sudoeste bonaerense, ordenados de acuerdo al valor absoluto decreciente del coeficiente de correlación con el $1^{\circ} \mathrm{CP} .\left(^{*}\right)$ Especies exóticas.

Table 1. Correlation coefficients between species cover and the first three PCA components in grassland fragments surrounded by a pine plantation, and controls of continuous grasslands of different area in coastal dunes of southwestern Buenos Aires Province, ordered according to the decreasing absolute value of correlation coefficient with the $1^{\circ}$ PC. $\left(^{*}\right)$ Exotic species.

\begin{tabular}{|c|c|c|c|}
\hline Especie & $1^{\circ} \mathrm{CP}$ & $2^{\circ} \mathrm{CP}$ & $3^{\circ} \mathrm{CP}$ \\
\hline essaria absinthioides (Ta) & 0,965 & $-0,141$ & 0,174 \\
\hline iliensis (Ib) & $-0,498$ & $-0,527$ & 0,645 \\
\hline rsium vulgare $(\mathrm{Cv})\left(^{*}\right)$ & 0,361 & $-0,179$ & $-0,073$ \\
\hline idago chilensis (Sc) & $-0,361$ & 0,282 & $-0,152$ \\
\hline enothera mollissima (Om) & 0,235 & $-0,218$ & $-0,095$ \\
\hline ) & $-0,234$ & $-0,105$ & 0,249 \\
\hline ata $(\mathrm{Hr})$ & 0,23 & $-0,087$ & $-0,137$ \\
\hline$T_{1}$ & $-0,224$ & $-0,327$ & 0,333 \\
\hline & 0,212 & $-0,044$ & $-0,05$ \\
\hline$y^{2}$ & $-0,195$ & $-0,06$ & 0,303 \\
\hline & $-0,173$ & $-0,023$ & $-0,231$ \\
\hline Amb & $-0,158$ & $-0,41$ & $-0,706$ \\
\hline & $-0,137$ & $-0,234$ & $-0,14$ \\
\hline & 0,134 & $-0,357$ & $-0,192$ \\
\hline$(*)$ & 0,132 & $-0,106$ & 0,071 \\
\hline & 0,129 & $-0,19$ & $-0,457$ \\
\hline$S c$ & $-0,119$ & 0,244 & 0,084 \\
\hline & $-0,084$ & 0,923 & 0,189 \\
\hline (1) 1 & $-0,081$ & 0,069 & $-0,376$ \\
\hline $\mathrm{Ce}^{\prime}$ & $-0,08$ & 0,445 & 0,028 \\
\hline & $-0,08$ & 0,75 & 0,05 \\
\hline Sonch & $-0,069$ & $-0,139$ & $-0,105$ \\
\hline & $-0,065$ & $-0,132$ & $-0,111$ \\
\hline$s$ (IMp) & $-0,065$ & 0,448 & $-0,033$ \\
\hline & $-0,048$ & 0,365 & 0,014 \\
\hline Ero & $-0,045$ & $-0,1$ & $-0,10$ \\
\hline ifolia (Bg) & $-0,037$ & 0,035 & $-0,157$ \\
\hline & -028 & $-0,478$ & 0,125 \\
\hline Sen & $-0,022$ & $-0,251$ & 20 \\
\hline
\end{tabular}

separó los controles de pastizal continuo de menor superficie de los fragmentos también más pequeños (agrupados en el primer y el cuarto cuadrante, respectivamente). Panicum urvilleanum, Aristida spegazzinii, Margyricarpus pinnatus, Cenchrus incertus, Senecio filaginoides, Solidago chilensis y Schizachyrium plumigerum, todas ellas nativas, predominaron en los controles de menor tamaño, mientras que Imperata brasiliensis, Cortaderia selloana, Ambrosia tenuifolia, Juncus acutus, Eragrostis airoides y la exótica Senecio madagascariensis lo hicieron en los fragmentos menores. El tercer componente (16.70\% de la varianza), por su parte, separó los fragmentos de mayor superficie de los controles también mayores. Las especies nativas Imperata brasiliensis, Eragrostis airoides, Achyrocline satureioides y Pseudognaphalium leucopeplum predominaron en los controles de mayor tamaño, mientras que en los fragmentos mayores se destacaron Ambrosia tenuifolia, Cyperus reflexus, Hydrocotyle bonariensis y Schoenoplectus americanus, también nativas (Tabla 1, Figura 5b).

\section{DISCUSIÓN}

Los resultados de este estudio permiten mostrar cómo la forestación con especies exóticas impacta sobre las comunidades vegetales de las dunas litorales del pastizal pampeano, fragmentando el ecosistema costero. Los fragmentos de pastizal rodeados por la matriz forestal evidenciaron modificaciones en la composición de la 
vegetación y, en particular, un aumento en el número de especies exóticas que resultó particularmente notorio en los remanentes de menor tamaño.

Pudimos detectar una disminución en el número de especies presentes a medida que se reduce la superficie de las unidades muestrales y este efecto se produjo tanto en los fragmentos rodeados por la matriz forestal como en los sitios de pastizal continuo. Este resultado es consistente con la visión clásica de los efectos de la fragmentación basados en las ideas de la teoría de biogeografía de islas (Preston 1962; MacArthur and Wilson 1967), pero contrasta con los resultados de un trabajo previo sobre fragmentación en pastizales de la Pampa Austral en el que no se detectaron efectos del área sobre la riqueza de especies vegetales (Herrera and Laterra 2007). Es posible que la diferencia entre nuestro trabajo y el de estos autores se deba a que se desarrollaron sobre escalas espaciales diferentes. Mientras el trabajo citado evaluó fragmentos cuya superficie varió entre 10 y más de 100 ha, nuestro análisis abarcó un rango de 0.05 a 2.5 ha. No pueden descartarse, sin embargo, otros factores que podrían ser responsables de esta diferencia, como la forma de los fragmentos, la distancia que los separa, su historia de uso o el tipo de comunidades vegetales afectadas por el proceso de fragmentación.

Pese a que la forma de los fragmentos varió en un rango amplio, no se observaron efectos sobre la riqueza de especies nativas y exóticas, ni sobre la diversidad de especies. Se espera que los fragmentos con formas más alejadas de la circular reciban un mayor efecto borde (Saunders et al. 1991). En nuestro caso, el hecho de que todos los fragmentos considerados fueron pequeños, podría resultar en que el efecto del contexto, por ejemplo en cuanto a la colonización por especies exóticas invasoras, afecte con intensidad a la totalidad de los fragmentos, independientemente de su relación perímetro/superficie.

La magnitud de la reducción en la riqueza de especies nativas y en la diversidad ante disminuciones equivalentes en el área censada resultó similar para los remanentes de pastizal rodeados por la forestación, respecto de las parcelas de pastizal continuo, lo que a priori podría tomarse como una evidencia de que el empobrecimiento biológico depende fundamentalmente del tamaño de las unidades de análisis, más allá del contexto ambiental. Esta preponderancia del efecto de la superficie ya había sido advertida por Haila (2002), sin embargo, las alteraciones que pueden atribuirse al contexto ambiental sí aparecen cuando se pone atención en áreas especialmente pequeñas o en grupos particulares de especies (Davies et al. 2001; Rosch et al. 2013). Así, las áreas menores inmersas en la matriz forestal mostraron caídas significativas en el número de plantas nativas cuando se las comparó con superficies equivalentes de pastizal continuo. La riqueza de plantas exóticas también se incrementó con la superficie de las unidades muestrales, aunque este aumento fue más marcado en el caso de los controles rodeados por pastizal. El número de especies exóticas en fragmentos rodeados por la plantación, por su parte, pareció experimentar una respuesta menos marcada respecto de la superficie, con valores elevados ya desde las áreas menores que resultaron significativamente mayores a los de las áreas continuas en el rango de 0.05 a 0.1 ha. El contexto ambiental podría, en este caso, condicionar tanto la llegada y establecimiento de las especies, como su persistencia bajo las condiciones locales. Las especies exóticas típicamente se comportan de manera oportunista y muchas de ellas se ven favorecidas por cambios en las condiciones ambientales generadas por la actividad humana (Davis et al. 2000; Gross et al. 2005; MacDougall and Turkington 2005; Dechoum et al. 2015). La matriz forestal que rodea a los fragmentos de pastizal podría modificar parámetros ambientales tales como la temperatura y la humedad, así como influir sobre los mecanismos de dispersión de propágulos (Amodeo et al. 2017; Marozas 2014), generando condiciones particularmente apropiadas para un conjunto de plantas exóticas en todo el rango de tamaños analizado (Lara and Gandini 2014). En concordancia con esto, la especie exótica Senecio madagascariensis se asoció de manera consistente con los remanentes de pastizal de menor tamaño y estuvo prácticamente ausente en los controles de pastizal continuo de superficie equivalente. Algo similar sucedió con Ambrosia tenuifolia, una especie nativa pero frecuentemente asociada a condiciones de disturbio (Insausti and Soriano 1982), que resultó dominante en alguno de los fragmentos rodeados por pinos, con coberturas superiores al $50 \%$, pero estuvo mucho menos representada en los controles de pastizal, sin superar el 10\% de cobertura en todo el rango de superficies considerado. 
Otra diferencia interesante en la composición de las comunidades vegetales de fragmentos rodeados por pinos, respecto de las áreas de pastizal continuo, es la mayor representación en los primeros de especies características de suelos bajos y húmedos, tales como Cyperus reflexus, Hydrocotyle bonariensis, Schoenoplectus americanus, Cortaderia selloana y Juncus acutus (Celsi and Monserrat 2008). Stevenson y Rodríguez (2008) consideran que, en ambientes fragmentados, la transición abrupta entre el paisaje original y la matriz resulta en cambios en los procesos bióticos (dispersión, polinización, etc.) y abióticos (radiación, viento, temperatura, humedad) que determinarán la composición de especies de la comunidad. Esas modificaciones pueden ser más marcadas en aquellas situaciones donde la matriz posee un tipo de vegetación estructuralmente diferente a la del ambiente natural (Ries et al. 2004; Herrera 2011; Bieringer et al. 2013; Marozas 2014), tal como es el caso en nuestra área de estudio. Es posible que allí los efectos de sombreamiento y de atenuación del viento producidos por la plantación circundante generen condiciones apropiadas para el establecimiento y persistencia de las especies higrófilas mencionadas. No podemos descartar, sin embargo, que la mayor representación de este tipo de vegetación en los fragmentos rodeados por la forestación responda a una decisión original de evitar las áreas más húmedas para la plantación.

Los resultados obtenidos en este trabajo coinciden con las conclusiones de otros autores: la riqueza de especies por sí sola puede confundir los efectos de la fragmentación, los efectos de este proceso son mayores a menor cantidad de hábitat, y el número de especies en sistemas fragmentados puede mantenerse en niveles elevados o incluso aumentar respecto de las áreas continuas por la presencia de especies exóticas o nativas oportunistas, aún a expensas de la retracción de taxones vulnerables (Saunders et al. 1991; Halffter and Moreno 2005; Rosch et al. 2013). Alternativamente, la composición específica resulta un indicador más preciso de las alteraciones asociadas a la fragmentación del pastizal por las plantaciones, tal como indican estudios realizados en ambientes forestales de Australia (Davies et al. 2001) o en pastizales de la Pampa Austral (Herrera and Laterra 2007). El análisis de los efectos de la fragmentación sobre la composición de las comunidades permite, además, plantear hipótesis acerca de factores particulares que podrían explicar la presencia o abundancia de determinados taxones, generando predicciones acerca de sus posibles efectos sobre otras especies con características similares, como se menciona previamente respecto de las variaciones en la abundancia de especies higrófilas en el área de estudio. Más aún, las variaciones en la representación de ciertos grupos pueden orientar trabajos dirigidos a evaluar el efecto de la fragmentación sobre procesos ecológicos tales como la polinización, dispersión, germinación, establecimiento y herbivoría, entre otros (Stevenson and Rodríguez 2008; Maguire et al. 2016).

Los resultados de este estudio refuerzan la importancia de conservar áreas continuas de vegetación como requisito para mantener la composición de las comunidades vegetales de las dunas litorales del ecosistema de pastizal pampeano y prevenir procesos de colonización por especies exóticas invasoras. Los fragmentos de 2.5 ha parecen exhibir una buena capacidad para resistir los procesos de invasión, mostrando niveles de riqueza de especies exóticas equivalentes a los de áreas de pastizal continuo de igual superficie, pudiendo considerarse este como un tamaño mínimo inicial para la conservación de fragmentos de pastizal psamófilo. Sin embargo, y considerando que el escenario más probable es el de un aumento de los procesos de fragmentación en este tipo de ambientes, la gestión sostenible de pastizales fragmentados dependerá de una profunda comprensión de los procesos y servicios ecosistémicos más afectados por este fenómeno, y el análisis de los factores responsables de los cambios en la composición de especies será un factor sensible para eso.

Agradecimientos. Agradecemos a los productores que permitieron realizar este estudio en sus establecimientos, a M. G. Kittler por su colaboración en las tareas de campo, y a M. R. Amodeo por sus sugerencias estadísticas. Este trabajo se realizó mediante el apoyo del Consejo Nacional de Investigaciones Científicas y Técnicas (CONICET). 


\section{REFERENCIAS}

Amodeo, M. R., B. Vázquez, and S. M. Zalba. 2017. Generalist dispersers promote germination of an alien fleshy-fruited tree invading natural grasslands. PLoS ONE 12:e0172423.

Azpiroz, A. B., J. P. Isacch, R. A. Dias, A. S. Di Giacomo, C. Suertegaray Fontana, and C. Morales Palarea. 2012. Ecology and conservation of grassland birds in southeastern South America: a review. Journal of Field Ornithology 83:217246.

Baldi, G., J. P. Guerschman, and J. M. Paruelo. 2006. Characterizing fragmentation in temperate South America grasslands. Agriculture, Ecosystems and Environment 116:197-208.

Barnes, A. D., R. M. Emberson, H. M. Chapman, F. Krell, and R. K. Didham. 2014. Matrix habitat restoration alters dung beetle species responses across tropical forest edges. Biological Conservation 170:28-37.

Begon, M., C. R. Townsend, and J. L. Harper. 2006. Ecology: From Individuals to Ecosystems. Fourth edition. Oxford : Blackwell science.

Benitez, J. 1998. Impact of forest fragmentation on seedling abundance in a Tropical Rain Forest. Conservation Biology 12:380-389.

Bieringer, G., K. P Zulka, N. Milasowszky, and N. Sauberer. 2013. Edge effect of a pine plantation reduces dry grassland invertebrate species richness. Biodiversity and Conservation 22:2269-2283.

Bilenca, D., and F. Miñarro. 2004. Identificación de Áreas Valiosas de Pastizal (AVPs) en las Pampas y Campos de Argentina, Uruguay y sur de Brasil. Fundación Vida Silvestre Argentina, Buenos Aires, Argentina.

Brugnoli, E., S. Masciadri, and P. Muniz. 2009. Base de datos de especies exóticas e invasoras en Uruguay, un instrumento para la gestión ambiental y costera. Ecoplata, Montevideo, Uruguay.

Burkart, R. 2006. Las Áreas Protegidas de la Argentina. Páginas 399-403 en A. Brown, U. Martínez Ortíz, M. Acerbi and J. Corcuera (eds.). La Situación Ambiental Argentina 2005. Fundación Vida Silvestre Argentina, Buenos Aires, Argentina.

Cabrera, A. L. 1963-1970. Flora de la Provincia de Buenos Aires. Colección Científica INTA, Buenos Aires, Argentina.

Cabrera, A.L. 1971. Fitogeografía de la República Argentina. Boletín de la Sociedad Argentina de Botánica 14:1-42.

Cairo, S. L., and S. M. Zalba. 2007. Effects of habitat fragmentation by a paved road on a population of endemic toads in the Argentine Pampas. Amphibia-Reptilia 28:377-385.

Campo de Ferreras, A., A. Capelli de Steffens, and P. Diez. 2004. El clima del Suroeste Bonaerense. Universidad Nacional del Sur, Bahía Blanca, Buenos Aires, Argentina.

Campo, J., A. Bacigalupe, B. Costa, and G. Pistone. 1999. Conservación y restauración del matorral psamófilo. Documentos de Trabajo 20. PROBIDES, Rocha, Uruguay.

Celsi, C. E., and A. L. Monserrat. 2006. Zona Costera del Partido de Coronel Dorrego: Por qué es conveniente proteger el paisaje natural de Marisol. Informe. Fundación de Historia Natural Félix de Azara, Buenos Aires, Argentina.

Celsi, C. E., and A. L. Monserrat. 2008. La vegetación dunícola en el frente costero de la Pampa Austral (Partido de Coronel Dorrego, Buenos Aires). Multequina 17:73-92.

Costanza, R., R. D'arget, R. Groot, S. Faber, M. Grasso, B. Hannon, K. Limburg, S. Naeem, T. V. O’Neill, J. Paruelo, R. Sutton, and M. Van den Belt. 1997. The value of the world's ecosystem services and natural capital. Nature 387: 253-260.

Cuevas, Y. A., and S. M. Zalba. 2009. Control de pinos invasores en el parque provincial Ernesto Tornquist (Buenos Aires): áreas prioritarias y análisis de costos. BioScriba 2:76-89.

Dadon, J. R. 2002. El impacto del turismo sobre los recursos naturales costeros en la costa pampeana. Páginas 101121 en J. R. Dadon and S. D. Matteucci (eds.). Zona Costera de la Pampa Argentina. Lugar Editorial, Buenos Aires, Argentina.

Davies, K. F., B. A. Melbuorne, and C. R. Margules. 2001. Effects of within- and between-patch processes on community dynamics in a fragmentation experiment. Ecology 82:1830-1846.

Davis, M. A., J. P. Grime, and K. Thompson. 2000. Fluctuating resources in plant communities: a general theory of invasibility. Journal of Ecology 88:528-534.

Dechoum, M. S., R. R. Zenni, T. T. Castellani, S. M. Zalba, and M. Rejmánek. 2015. Invasions across secondary forest successional stages: effects of local plant community, soil, litter, and herbivory on Hovenia dulcis seed germination and seedling establishment. Plant Ecology 216:823-833.

Fahrig, L. 2003. Effects of habitat fragmentation on biodiversity. Annual Review of Ecology, Evolution, and Systematics 34:487-515.

Fonseca, C., D. L. Guadagnin, C. Emer, S. Masciadri, P. Germain, and S. M. Zalba. 2013. Invasive alien plants in the Pampas grasslands: a tri-national cooperation challenge. Biological Invasions 15:1751-1763.

Gross, K. L., G. G. Mittelbach, and H. L. Reynolds. 2005. Grassland invasibility and diversity: responses to nutrients, seed input, and disturbance. Ecology 86:476-486.

Guadagnin, D. L., S. M. Zalba, B. Costa Gorriz, C. R. Fonseca, A. J. Nebbia, Y. A. Cuevas, C. Emer, P. Germain, E. Wendland, L. F. C. Perello, M. C. S. Bastos, C. Sanhueza, S. Masciadri Bálsamo, and A. E. de Villalobos. 2009. Árvores e arbustos exóticos invasores no Bioma Pampa - questões ecológicas, culturais e socioeconômicas de um desafio crescente. Páginas 300-316 en V. De Patta Pillar, S. C. Müller, Z. M de Souza and A. V. Ávila Jacques (eds.). Campos 
Sulinos: conservação e uso sustentable da biodiversidade. Ministerio do Meio Ambiente, Brasilia, Brasil.

Haddad, N. M., L. A. Brudvig, J. Clobert, K. F. Davies, A. Gonzalez, R. D. Holt, T. E. Lovejoy, J. O. Sexton, M. P. Austin, C. D. Collins, W. M. Cook, E. I. Damschen, R. M. Ewers, B. L. Foster, C. N. Jenkins, A. J. King, W. F. Laurance, D. J. Levey, C. R. Margules, B. A. Melbourne, A. O. Nicholls, J. L. Orrock, D. X. Song, and J. R. Townshend. 2015. Habitat fragmentation and its lasting impact on Earth's ecosystems. Science Advances 1:e1500052.

Haila, Y. 2002. A conceptual genealogy of fragmentation research: from island biogeography to landscape ecology. Ecological Applications 12:321-334.

Halffter, G., and C. Moreno. 2005. Significado biológico de las diversidades alfa, beta y gamma. Páginas 5-18 en G. Halffter, J. Soberon, P. Koleff and A. Melic (eds.). Sobre Diversidad Biológica: el Significado de las Diversidades Alfa, Beta y Gamma. Monografías Tercer Milenio, Zaragoza, España.

Herrera, J. M. 2011. El papel de la matriz en el mantenimiento de la biodiversidad en hábitats fragmentados. De la teoría ecológica al desarrollo de estrategias de conservación. Ecosistemas 20:21-34.

Herrera, L., and P. Laterra. 2007. Relaciones entre la riqueza y la composición florística con el tamaño de fragmentos de pastizales en la Pampa Austral, Argentina. Páginas 387-396 en S. D. Matteucci (ed.). Panorama de la Ecología de Paisajes en Argentina y Países Sudamericanos. Ediciones INTA, Buenos Aires, Argentina.

Hoekstra, J. M., T. M. Boucher, T. H. Ricketts, and C. Roberts. 2005. Confronting a biome crisis: global disparities of habitat loss and protection. Ecology Letters 8:23-29.

Insausti, P., and A. Soriano. 1982. Comportamiento de las semillas de Ambrosia tenuifolia (Altamisa) en un pastizal de la Depresión del Salado. Revista de la Facultad de Agronomia 3: 75-80.

Jobbágy, E. G., M. Vasallo, K. A. Farley, G. Piñeiro, M. F. Garbulsky, M. D. Nosetto, R. B. Jackson, and J. M. Paruelo. 2006. Forestación en pastizales: hacia una visión integral de sus oportunidades y costos ecológicos. Agrociencias 10: 109-124.

Kittlein, M. J., M. S. Mora, A. I. Vassallo, F. de Durana, M. G. Ricciardulli, and F. R. Tizón. 2004. Dunas del sureste bonaerense. Páginas 76-77 en D. Bilenca and F. Miñarro (eds.). Identificación de Áreas Valiosas de Pastizal (AVPs) en las Pampas y Campos de Argentina, Uruguay y sur de Brasil. Fundación Vida Silvestre, Buenos Aires, Argentina.

Krause, B., H. Culmsee, K. Wesche, and C. Leuschner. 2015. Historical and recent fragmentation of temperate floodplain grasslands: Do patch size and distance affect the richness of characteristic wet meadow plant species? Folia Geobotanica 50:253-266.

Lara, B., and M. Gandini. 2011. Biogeografía de islas en fragmentos de pajonal del Paisaje Ariel (Azul, Buenos Aires, Argentina). Revista de la Asociación Argentina de Ecología del Paisaje 2:1-8.

Lara, B., and M. Gandini. 2014. Análisis de la fragmentación de pastizales en la Pampa Deprimida (Argentina). SEMIÁRIDA Revista de la Facultad de Agronomía UNLPam 24:21-30.

MacArthur, R. H., and E. O. Wilson. 1967. The theory of island biogeography. Princeton University Press, Princeton, New Jersey, USA.

MacDougall, A. S, and R. Turkington. 2005. Are invasive species the drivers or passengers of change in degraded ecosystems? Ecology 86: 42-55.

Maguire, D. Y., C. M. Buddle, and E. M. Bennett. 2016. Within and Among Patch Variability in Patterns of Insect Herbivory Across a Fragmented Forest Landscape. PLoS ONE 11:e150843.

Marozas, V. 2014. Effect of the coniferous forest-grassland edge on ground vegetation in the mixed European forest zone, Lithuania. Dendrobiology 71:15-22.

Metzger, J. P. 2000. Tree Functional Group Richness and Landscape Structure in a Brazilian Tropical Fragmented Landscape.

Ecological Applications 10:1147-1161.

Monserrat, A. L., and C. E. Celsi. 2009. Análisis regional de la costa pampeana austral en el marco del sistema de áreas protegidas y caracterización de un área clave como reserva, en el partido de Coronel Dorrego. Bioscriba 2:1-23.

Mueller Dombois, D., and E. Ellenberg. 1974. Aims and Methods of Vegetation Ecology. John Wiley and Sons, New York, USA.

Nebbia, A. J., and S. M. Zalba. 2007. Designing nature reserves: traditional criteria may act as misleading indicators of quality. Biodiversity and Conservation 16:223-233.

Picasso, G., and E. Rodríguez. 2008. Bañados del Este. Páginas 289-294 en E. Schüttler and C. S. Karez (eds.). Especies exóticas invasoras en las Reservas de Biosfera de América Latina y el Caribe. UNESCO, Montevideo, Uruguay.

Preston, F. W. 1962. The canonical distribution of commonness and rarity. Ecology 43:185-215, 410-432.

Raffaele, E., and T. Schlichter. 2000. Efectos de las plantaciones de pino ponderosa sobre la heterogeneidad de micrositios en estepas del noroeste patagónico. Ecología Austral 10:151-158.

Ries, L., R. J. Fletcher, J. Battin, and T. D. Sisk. 2004. Ecological responses to habitat edges: mechanisms, models and variability explained. Annual Review of Ecology, Evolution, and Systematics 35:491-522.

Rosch, V., T. Tscharntke, C. Scherber, and P. Batáry. 2013. Landscape composition, connectivity and fragment size drive effects of grassland fragmentation on insect communities. Journal of Applied Ecology 50:387-394.

Saunders, D. A., R. J. Hobbs, and C. R. Margules. 1991. Biological consequences of ecosystem fragmentation: A Review. Conservation Biology 5:18-32.

Schmidt, B. C., and J. Roland. 2006. Moth Diversity in a Fragmented Habitat: Importance of Functional Groups and Landscape Scale in the Boreal Forest. Annals of the Entomological Society of America 99:1110-1120. 
Simberloff, D., M. A. Nuñez, N. J. Ledgard, A. Pauchard, D. M. Richardson, M. Sarasola, B. Vanwilgen, S. M. Zalba, R. Zenni, R. Bustamante, E. Peña, and S. R. Ziller. 2010. Spread and impact of introduced conifers in South America: Lessons from other southern hemisphere regions. Austral Ecology 35:489-504.

Stevenson, P. R., and M. E. Rodríguez. 2008. Determinantes de la composición florística y efecto de borde en un fragmento de bosque en el Guaviare, Amazonia colombiana. Colombia Forestal 11:5-18.

Suttie, J. M., S. G. Reynolds, and C. Batello. 2005. Grasslands of the world. Food and Agriculture Organization of the United Nations (FAO), Rome, Italy.

Thiollay, J. M. 1988. Forest Fragmentation and the Conservation of Raptors: A Survey on the Island of Java. Biological Conservation 44:229-250.

Wilson, M. C., X. Y. Chen, R. T. Corlett, R. K. Didham, P. Ding, R. D. Holt, M. Holyoak, G. Hu, A. C. Hughes, L. Jiang, W. F. Laurance, J. Liu, S. L. Pimm, S. K. Robinson, S. E. Russo, X. Si, D. S. Wilcove, J. Wu, and M. Yu. 2016. Habitat fragmentation and biodiversity conservation: key findings and future challenges. Landscape Ecology 31:219-227.

With, K. A. 2004. Assessing the risk of invasive spread in fragmented landscapes. Risk Analysis 24:803-815.

Yates, E. D., D. F. Levia, and C. L. Williams. 2004. Recruitment of three non-native invasive plants intoa fragmented forest in southern Illinois. Forest Ecology and Management 190:119-130.

Zalba, S. M., and A. J. Nebbia. 1999. Neosparton darwinii (Verbenaceae) a restricted endemic species. Is it also endangered? Biodiversity and Conservation 8:1585-1593.

Zalba, S. M., and C. B. Villamil. 2002. Woody plant invasion in relictual grasslands. Biological Invasions 4:55-72.

Zuloaga, F. O., O. Morrone, and M. J. Belgrano. 2008. Catálogo de las Plantas Vasculares del Cono Sur. Monographs in Systematic Botany from the Missouri Botanical Garden, St. Louis, Missouri, USA. 